\title{
Um Estudo Terciário Sobre o Ensino de Computação no Brasil
}

Leo Natan Paschoal, Universidade de São Paulo, paschoalln@usp.br

Pedro Henrique Dias Valle, Universidade de São Paulo, pedrohenriquevalle@usp.br Silvana M. Melo, Universidade Federal da Grande Dourados, silvanamelo@ufgd.edu.br

Resumo: O ensino de computação tem ganhado espaço em conferências e periódicos brasileiros que envolvem tecnologias educacionais. Nesse contexto, têm sido discutidas abordagens e mecanismos para apoiar (i) iniciativas que visam desenvolver o pensamento computacional, (ii) movimentos que visam promover mulheres na computação e (iii) problemas que emergem no contexto de ensino de disciplinas de Computação. Nessa perspectiva, estudos secundários vêm sendo produzidos, com o propósito de sintetizar as contribuições já estabelecidas. Apesar disso, não há um panorama sobre quais os tópicos sobre o ensino de Computação já foram investigados, bem como os problemas inerentes a esses tópicos e suas soluções. Nesse sentido, este artigo aborda um estudo terciário, visando contribuir com essa temática, por meio de uma análise das evidências sobre os tópicos abordados em ensino de Computação, os principais problemas e soluções propostas.

Palavras-chave: Ensino de Computação, Revisão Sistemática, Estudo Terciário.

\section{A Tertiary Study on Computing Education in Brazil}

Abstract: Computing education has gained space in Brazilian conferences and journals involving educational technologies. In this context, approaches and mechanisms have been discussed to support (i) initiatives that aim to develop computational thinking, (ii) efforts to promote women in computing, and (iii) problems that emerge in the context of Computer teaching subjects. In this perspective, secondary studies have been produced, to synthesize the contributions already established. Nevertheless, there is no overview on which topics on Computing teaching have already been investigated, as well as the problems inherent in these topics and their solutions. In this direction, this article addresses a tertiary study, with the pourpose of contributing to this theme, through an evidence analysis on Computer topics, the problems, and proposed solutions.

Keywords: Computing Education, Systematic Review, Tertiary Study.

\section{Introdução}

Desde o surgimento do computador, o aprendizado de computação tem ganhado cada vez mais espaço na sociedade. Isso pode ser um reflexo sobre o entendimento que as comunidades científicas e professores possuem em relação à necessidade de formar alunos com habilidades que superam a assimilação e manuseio de sistemas de software. Atualmente, há estudos que abordam a necessidade de formar indivíduos com competências que possibilitam o entendimento concreto sobre problemas que requerem soluções não triviais e que esses indivíduos sejam capazes de estabelecer contribuições para resolvê-los (ZHANG; NOURI, 2019).

Aliado a isso, a computação tem promovido um grande impacto em outras áreas, dado que problemas complexos de áreas que não possuem origens na computação têm tentado estabelecer soluções com uma perspectiva de computação (BRACKMANN, 2017). Assim, o aprendizado dessa temática se faz necessário para os sujeitos que fazem parte da sociedade atual. Besta forma, é possível assumir que o aprendizado de computacão para DOI: 
esses indivíduos é tão importante quanto o conhecimento sobre matemática, física, biologia, linguagens, dentre outros (BRACKMANN, 2017). Consequentemente, iniciativas que favoreçam o desenvolvimento do pensamento computacional estão se tornando presentes nos diferentes municípios, estados, países e continentes.

Ao passo que há o surgimento de iniciativas para o desenvolvimento do pensamento computacional, é preciso compreender como aplicá-las de modo eficaz, como adaptá-las em contextos e culturas específicas, como organizar os projetos políticos pedagógicos das escolas de modo a integrar essas abordagens, como as escolas que possuem estruturas desiguais podem acompanhar e fazer parte dessas iniciativas. Além disso, é preciso obter um entendimento sobre como conceber ferramentas e mecanismos de apoio ao ensino que possibilitam a promoção e desenvolvimento dessas iniciativas em contextos educacionais tão diversificados.

Os artigos científicos têm sido usados como mecanismo da sociedade para divulgar os conhecimentos que estão sendo obtidos nessas iniciativas (ZHANG; NOURI, 2019). Eles podem ajudar outros professores, cientistas e organizações a implementar novas iniciativas em lugares que ainda não foram contemplados por tais projetos. As lições aprendidas que são descritas no decorrer desses estudos podem ser usadas para inspirar mudanças em iniciativas vigentes que necessitam de melhorias. Portanto, esses estudos são importantes para a comunidade e se organizados de modo sistemático, podem contribuir ainda mais com a temática, no sentido de oferecer algumas evidências, tais como: os problemas com maior quantidade de incidência nessas iniciativas, as soluções comumente definidas para solucionar tais problemas, dentre outros.

Esses artigos científicos normalmente são publicados em veículos de divulgação científica sobre educação em computação ou informática na educação. Além desse tipo de estudo, também se fazem presentes trabalhos que discutem problemas e soluções no contexto de ensino de cursos de graduação em computação (SOUZA; BATISTA; BARBOSA, 2016), dado que o ensino de alguns assuntos de computação tem recebido ampla atenção por parte de professores de computação. Nesse contexto, os problemas abordados são associados a uma disciplina ou um tópico de uma disciplina do curso (e.g., programação) e são discutidas soluções para tais problemas (HOLANDA; FREIRE; COUTINHO, 2019; LIMA et al. 2019). Esses problemas geralmente envolvem soluções que fazem uso de experimentação de abordagens pedagógicas (e.g., metodologias ativas) e/ou o estabelecimento de mecanismos de apoio ao ensino (e.g., jogos educacionais, objetos de aprendizagem, laboratórios de aprendizagem, dentre outros).

Além das problemáticas associadas as disciplinas, os cursos de computação têm atraído um número crescente de alunos (HAO et al., 2019). Apesar disso, as vagas desses cursos têm sido preenchidas quase que exclusivamente por homens. Relatórios têm reportado que as mulheres tem perdido espaço na computação (RIDEL et al., 2018). Nessa perspectiva, estudos têm sido produzidos para compreender esse fenômeno e alguns movimentos têm sido articulados para reverter essa situação.

Os movimentos para despertar o interesse de meninas por computação envolvem ações dedicadas a estudantes do ensino fundamental e médio, visando ensiná-las programação, tecnologias e empreendedorismo (RIDEL et al., 2018). Além do ensino de tais conteúdos, alguns movimentos englobam palestras e debates com profissionais de empresas de tecnologia, demonstrações de exemplos para estimulá-las a ingressar em cursos nesse campo (TRIDICO et al., 2018). 
Similarmente às iniciativas de desenvolvimento de pensamento computacional, os movimentos que visam retomar o espaço das mulheres em cursos de computação também são tópicos de interesse em eventos e periódicos com foco na divulgação de experiências e lições aprendidas sobre ensino de computação. Diante disso, relatos de experiência têm promovido discussões sobre essas ações, descrevendo como elas são implantadas, os desafios de implantá-las, dentre outros. Esse tipo trabalho pode ser usado como base por grupos de trabalho que estão interessados em desenvolver esses movimentos.

Nessa perspectiva, um panorama sobre esses trabalhos também pode ajudar a contribuir ainda mais com o surgimento desse tipo de movimento, dado que tem a capacidade de sintetizar as ações e responder perguntas que transcrevam as dúvidas de profissionais que têm a intenção de promover esses movimentos em suas organizações, tais como: quais os maiores desafios em desenvolver esses movimentos? ; quais ações promovem mais engajamento entre as estudantes? ; quais os principais resultados obtidos por meio dessas iniciativas ? ; quais entidades e organizações podem ajudar a instituir tais movimentos ? ; dentre outros.

A síntese de artigos científicos tem sido amplamente realizada no contexto de ensino de computação, por meio de métodos de revisão sistemática de literatura (KITCHENHAM et al., 2010). Em particular, no Brasil existem diversos estudos (secundários) que propuseram mapear a temática de ensino de computação. No entanto, não há um entendimento geral sobre o que esses estudos tem de fato sintetizado. Nesse sentido, algumas questões precisam ainda ser discutidas, a saber: (i) quais os tópicos sobre o ensino de computação já possuem uma síntese produzida de modo sistemático? (ii) as sínteses têm abordado os problemas inerentes ao ensino de computação e as soluções pra esses problemas ou têm dado atenção a outros aspectos? .

Tendo como base o que foi descrito ao longo desta seção, este artigo emerge com o propósito de apresentar um estudo que buscou identificar evidências sobre quais os tópicos de computação já foram analisados sistematicamente pela comunidade, bem como os problemas e soluções relacionados a esses tópicos. Este estudo faz parte de um projeto maior no qual busca-se obter um panorama sobre os tópicos de ensino de computação já estudados, os problemas sobre esses tópicos que já foram resolvidos e aqueles que ainda precisam ser solucionados. Para tanto, foi conduzido um estudo terciário, um método que pode ser definido como uma revisão sistemática de estudos secundários usando a mesma metodologia da revisão sistemática (KITCHENHAM et al., 2010).

Para apresentar este estudo, o artigo foi organizado da seguinte maneira. A seção 2 descreve os procedimentos utilizados. Na Seção 3 são apresentados os resultados. Durante a Seção 4 são discutidas as limitações deste estudo. Por fim, a Seção 5 aborda as principais conclusões e indica alternativas à trabalhos futuros.

\section{Materiais e métodos}

Nesta seção, definiram-se as questões de pesquisa a serem esclarecidas, os critérios de inclusões e exclusão dos estudos e o processo de extração de dados.

\subsection{Questões de pesquisa}

Com o objetivo de conduzir um estudo terciário para sintetizar as evidências existentes sobre o ensino de computação, foram formuladas as seguintes questões de pesquisa (QP):

$\mathbf{Q P}_{1}$ : Quais os tópicos na área da computação têm sido investigados pelos estudos seV. 18 N cundários julho, 2020 nduzidos pela comunidade brasileira de informática na ed Reacão? DOI: 
$\mathbf{Q P}_{2}$ : Quais problemas relacionados ao processo de ensino-aprendizagem têm sido investigados no contexto do ensino de computação?

$\mathbf{Q P}_{3}$ : Quais soluções têm sido propostas no contexto de ensino de computação?

\subsection{Estratégias de busca}

O processo para busca foi conduzido via seleção manual para identificação de artigos publicados (disponíveis online). Para responder as questões de pesquisa foram consideradas nesse processo as publicações das principais conferências e periódicos brasileiros da área de ensino em computação, são eles: SBIE (Simpósio Brasileiro de Informática na Educação), WIE (Workshop de Informática na Escola), WEI (Workshop sobre Educação em Computação), WCBIE (Workshops do Congresso Brasileiro de Informática na Educação), RBIE (Revista Brasileira de Informática na Educação) e RENOTE (Revista Novas Tecnologias na Educação).

\subsection{Critérios de seleção}

A fim de conduzir a seleção dos estudos secundários, foram definidos os seguintes critérios de inclusão (CI) e exclusão (CE): $\mathbf{C I}_{1}$ Estudos que apresentam uma revisão sistemática da literatura sobre ensino de computação; $\mathbf{C E}_{1}$ Estudos que não apresentam uma revisão sistemática da literatura sobre ensino de computação; $\mathbf{C E}_{2}$ Estudos que embora apresentem uma revisão sistemática não abordam ensino de computação; $\mathbf{C E}_{3}$ Estudos em outra linguagem diferente de inglês e português.

\subsection{Extração de dados e estratégias de síntese}

Ao final da do processo de seleção, considerando os critérios de inclusão e exclusão apresentados, foram incluídos a este estudo terciário 36 estudos secundários que compõem o conjunto final de estudos sobre os quais a extração foi conduzida Artigos resultantes da busca foram analisados pelos revisores considerando: título; autores; ano de publicação; base de dados; tópico de computação; problemas e soluções propostas; ameaças à validade; contexto (nacional e internacional). Para tanto, foi definido um formulário de apoio a extração de dados

\subsection{Condução do estudo terciário}

O estudo foi conduzido durante o primeiro semestre de 2020. O processo de busca foi dividido em três etapas. Primeiramente foram realizadas buscas manuais nas conferências e periódicos definidos na Seção 2.2. Os estudos identificados na fase anterior foram analisados e apenas os que apresentavam uma revisão sistemática permaneceram. E por fim, na última fase foi realizada a leitura completa de cada um dos artigos e os dados foram extraídos individualmente por cada revisor. A Tabela 1 sumariza os resultados obtidos ao final do processo de seleção.

Tabela 1. Síntese dos resultados da busca por base de dados
\begin{tabular}{|l|c|c|c|c|c|c|c}
\hline Base de dados & RENOTE & RBIE & SBIE & WEI & WIE & WCBIE & Total \\
\hline Estudos secundários & 7 & 2 & 16 & 2 & 6 & 4 & 36 \\
\hline
\end{tabular}

Na Figura 1, é possível obter uma visão geral da distribuição dos estudos pelo território brasileiro baseado na localização das instituições de origem do autor principal de

\footnotetext{
${ }^{1} \mathrm{~A}$ lista completa dos estudos está disponível em: $<$ https://bit.ly/3eXWPmZ $>$.

$\mathrm{V}^{2}$ Disponível em: $18 \mathrm{~N}$ https:///bit.ly/30FSArd $>$.

RENOTE

DOI:
} 
cada revisão sistemática. No geral, foram identificados 11 estados que têm contribuído com estudos secundários, são eles: Goiás (5), Pernambuco (5) e Rio grande do Norte (5), Rio Grande do Sul (4), São Paulo (4), Paraíba (3), Santa Catarina (3), Rio de Janeiro (2), Amazonas (1), Maranhão (1), Paraná (1), Piauí (1), Roraima (1).

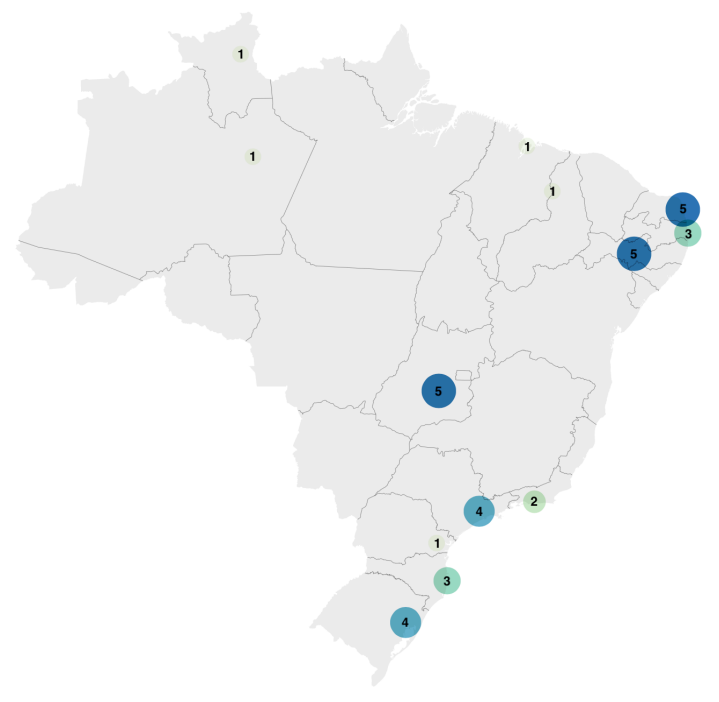

Figura 1. Distribuição por localização dos autores das revisões sistemáticas.

\subsection{Ameaças à validade}

Todo estudo baseado em evidência deve identificar possíveis ameaças para o nível de confiança dos resultados obtidos. Nesse sentido, as principais ameaças à validade deste estudo terciário são: exclusão de artigos relevantes e o uso de artigos com qualidade reduzida. A fim de reduzir a possibilidade de introdução de erros à pesquisa, as principais conferências na área de informática na educação e educação em computação do país foram consideradas. Além disso, os filtros feitos nos estudos encontrados foram efetuados com base no conhecimento de três revisores distintos.

\section{Resultados e discussão}

Nesta seção são apresentadas as evidências coletadas para responder as questões de pesquisa definidas no planejamento deste estudo, bem como uma breve discussão sobre os resultados obtidos.

\subsection{Visão geral dos estudos secundários}

Conforme descrito anteriormente, selecionaram-se 36 estudos secundários que investigaram o ensino de computação no Brasil para coletar evidências com o intuito de responder as questões de pesquisa propostas. Esses estudos foram publicados entre os anos de 2012 a 2019, com uma maior frequência nos últimos anos. Especificamente oito estudos secundários foram publicados no ano de 2019. Isso demonstra um forte interesse da comunidade em investigar o ensino de computação no Brasil e um grande esforço da comunidade em mapear as evidências de estudos primários nessa temática.

Grande parte dos estudos secundários utilizaram máquinas de buscas conhecidas no contexto de computação (i.e., IEEE Xplore, Scopus e ACM Digital Library) para identificar os estudos primários analisados por eles. Há também estudos que fizeram busca manuais nos principais congressos e periódicos da área, sendo eles: SBIE, WIE, WEI, SBGAMES (Simpósio Brasileiro de Games e Entretenimento Digital), WCBIE RENOTE e DOI: 
RBIE. Desse modo, observou-se que não há um padrão em utilizar uma estratégia de busca específica para identificar os estudos primários. Em geral, os estudos que investigaram o ensino de computação no Brasil foram publicados em congressos e periódicos no contexto nacional. No contexto deste trabalho, decidiu-se realizar a busca pelos estudos secundários de forma manual apenas em eventos e periódicos brasileiros.

Com relação ao idioma considerado, 21 estudos consideraram estudos primários em inglês e português, nove estudos incluíram apenas estudos primários em português, quatro estudos analisaram apenas estudos primários em inglês e somente um estudo considerou estudos primários em inglês, espanhol e português.

Os estudos primários foram selecionados em diferentes contextos, em especial 21 estudos secundários buscaram por estudos primários no contexto internacional e 15 estudos buscaram por estudos primários apenas no contexto nacional. Também foi verificado se os autores dos estudos secundários consideraram ameças à validade dos seus estudos. Como resultado, apenas 4 estudos secundários fizeram essa análise.

Por fim, apenas 17 estudos secundários discutiram lacunas em aberto no ensino de computação. Essas informações podem direcionar novas pesquisas na área, estabelecendo novas soluções para os problemas existentes no ensino de computação.

\subsection{Tópicos de computação}

A primeira questão de pesquisa teve como objetivo principal identificar os tópicos de computação que foram investigados por meio de estudos secundários pela comunidade brasileira no contexto de ensino de computação. Como resultado, identificaram-se sete tópicos diferentes, além de alguns estudos que investigaram abordagens para apoiar o ensino de computação de forma geral (classificado em disciplinas gerais de computação). A Tabela 2 sumariza os resultados identificados para a $R_{1}$.

Tabela 2. Tópicos de computação investigados pelos estudos secundá
\begin{tabular}{l|c}
\multicolumn{1}{c}{ Tópicos } & Quantidade \\
\hline Algoritmos e Programação & 22 \\
\hline Engenharia de software & 5 \\
\hline Disciplinas gerais de computação & 4 \\
\hline Estrutura de dados & 1 \\
\hline Robótica & 1 \\
\hline Programação paralela & 1 \\
\hline Programação orientada a objetos & 1 \\
\hline Redes de computadores & 1 \\
\hline Total & 36 \\
\hline
\end{tabular}

De forma geral, Algoritmos e Programação é o tópico mais investigado em estudos secundários pela comunidade brasileira no contexto de Ensino de computação. Aproximadamente, $61 \%$ dos estudos propuseram soluções e/ou investigaram problemas e desafios para este tópico. Isso pode estar relacionado com a complexidade para ensinar este tópico, uma vez diversos estudos têm reportado uma dificuldade por parte dos alunos em aprenderem tais conteúdos devido, principalmente, a dificuldade de raciocínio lógico, resolução de problemas e dificuldade em entender e/ou aplicar termos abstratos.

O tópico de Engenharia de Software foi o segundo tópico mais investigado de acordo com as ${ }_{0}$ evidências obtidas neste estudo, aproximadamente $14 \%$ dos estudos inyestigaram DOI: 
este tópico. Em particular, dois estudos investigaram soluções para o ensino de Teste de Software, o qual é um subtópico da área de Engenharia de Software. Enquanto que os tópicos de Estruturas de Dados, Robótica, Programação Paralela, Programação Orientada a Objetos, e Redes de Computadores foram investigados por apenas um estudo secundário cada. Em outra perspectiva, quatro estudos investigaram o ensino de computação de forma geral, sem aprofundar em um tópico especifico.

\subsection{Problemas no ensino de computação}

A segunda questão de pesquisa teve como objetivo identificar problemas recorrentes para o ensino de computação. Apenas 6 dos 36 estudos analisados investigaram problemas no ensino de computação. Esses problemas são recorrentes no ensino de Programação (SOUZA; BATISTA; BARBOSA, 2016; RODRIGUES; ARANHA; SILVA, 2018; HOLANDA; FREIRE; COUTINHO, 2019), Engenharia de software (LIMA et al., 2019), Programação Orientada a Objetos (RICHTER; BERNARDI; CORDENONSI, 2019) e disciplinas gerais de computação (ARMANDA; RODRIGUES; GARCIA, 2012). Os problemas identificados foram categorizados de acordo com os tópicos investigados.

- Algoritmos e Programação: (i) Incompatibilidade das atividades para promover o pensamento computacional com diferentes faixas etária; (ii) Inadequação de ambientes físicos para a realização das atividades, como a falta de computador e laboratórios; (iii) Evasão no decorrer da aplicação das abordagens e reprovação dos alunos; (iv) Tipo de abordagem que utiliza aulas puramente expositivas e exercícios esparsos; (v) Falta de motivação dos alunos; (vi) Ausência de mulheres nos cursos de computação; (vii) Ausência de ferramentas gratuitas; (viii) Dificuldades dos alunos relacionadas à aprendizagem de conceitos de programação; (iv) Dificuldade dos alunos durante a aplicação de conceitos de programação aprendidos; (x) Dificuldade dos alunos compreender programas; (xi) Dificuldade dos alunos em fatorar e refatorar de módulos, funções e classes; (xii) Dificuldades dos professores em ensinar os conceitos de programação, desenvolver materiais e exercícios de apoio, bem como avaliar os trabalhos de programação.

- Programação Orientada a Objetos: (i) Evasão no decorrer da aplicação das abordagens e reprovação dos alunos; (ii) Dificuldades dos alunos que estão relacionadas à aprendizagem de conceitos de programação; (iii) Dificuldades dos alunos em aplicar conceitos de programação aprendidos; (iv) Dificuldade dos alunos em compreender a abstração e/ou aplicar termos abstratos.

- Engenharia de Software: (i) Dificuldade dos alunos em aprender conceitos da área de engenharia de software; (ii) Desmotivação e desinteresse em aprender os conceitos da área, bem como seguir carreira com o papel de engenheiro de software; (iii) Ausência de habilidades interpessoais; (iv) Baixa aplicação da teoria aprendida em projetos reais; (v) Complexidade dos conteúdos abordados; (vi) Ausência de atividades discursivas e baixa relação entre o professor e o aluno.

- Tópicos gerais de computação: (i) Dificuldade na utilização de tecnologias; (ii) A forma como a avaliação dos alunos é conduzida; (iii) Falta de tempo para ensinar todos os conteúdos pretendidos.

A partir das evidências apresentadas anteriormente, pode-se observar que em alguns tópicos há uma carência de estudos secundários que investiguem quais são os problemas existentes no ensino de computação. Essa informação é essencial para entender quais os problemas já foram solucionados pela comunidade brasileira, e consequente, poderá ser utilizada para propor novas solução para os problemas ainda existentes. A partir disso, será possível formar profissionais qualificados para inserir no mercado de trabalho ${ }_{\text {RENOTE }}$ DOI: 


\subsection{Soluções para os problemas no ensino de computação}

A terceira questões de pesquisa teve como objetivo identificar soluções existentes para os problemas recorrentes no ensino de computação. A partir dos estudos analisados, identificaram-se 35 diferentes tipos de soluções, que foram agrupadas por tópicos.

- Algoritmos e Programação: (i) Metodologias ativas;(ii) Jogos educacionais; (iii) Gamificação; (iv) Software educacional;(v) Recursos multimídia; (vi) Ambientes de programação; (vii) Computação desplugada; (viii) Robótica; (iv) Simuladores educacionais; (x) Arquiteturas pedagógicas; (xi) Desenvolvimento Orientado por Testes; (xii) Residência de software; (xiii) Notações; (xiv) Feedback; (xv) Representações semânticas; (xvi) Sistemas tutores inteligentes; (xvii) Metáforas lúdicas; (xviii) Dojo; (xix) Sistemas de submissão de exercícios; (xx) Música e danças; (xxi) Brincadeiras e desafios; (xxii) Contextualização indisciplinas; (xxiii) Estudos de casos; (xxiv) Aprendizagem baseada em desafios.

- Robótica: (i) Kits de arduíno; (ii) Kit de robótica Lego.

- Estruturas de dados: (i) Jogos educacionais; (ii) Software educacional; (iii) Recursos multimídia.

- Engenharia de Software:(i) Metodologias ativas; (ii) Jogos educacionais; (iii) Gamificação; (iv) Software educacional; (v) Recursos multimídia; (vi) Ambientes de programação; (vii) Robótica; (viii) Simulador educacional; (ix) Aprendizagem Colaborativa e Desenvolvimento Orientado por Testes; (x) Tutoriais; (xi) Redes sociais; (xii) Residência de software; (xiii) Sistemas tutores inteligentes; (xiv) Metáforas lúdicas; (xv) Música e dança; (xvi) Inquiry-based learning; (xvii) Aplicativos para m-learning.

- Programação Paralela: (i) Metodologias ativas; (ii) Recursos multimídia; (iii) Aprendizagem colaborativa; (iv) Sistemas tutores inteligentes; (v) Metáforas lúdicas.

- Programação Orientada a Objetos: (i) Metodologias ativas; (ii) Jogos educacionais; (iii) Gamificação; (iv) Recursos multimídia; (v) Ambientes de programação; (vi) Scaffolding; (vii) Robótica.

- Tópicos Gerais: (i) Metodologias ativas; (ii) Jogos educacionais; (iii) Gamificação; (iv) Trabalhos em grupo; (v) Ambientes de programação; (vi) Ambientes virtuais; (vii) Computação desplugada; (viii) Robótica.

Os tópicos de Programação e Engenharia de Software são os tópicos mais investigados pelos estudos secundários, conforme apresentado na Seção 3.2, e consequentemente, a maior parte das soluções identificadas são aplicadas para apoiar o ensino desses conteúdos. Os estudos secundários que investigaram esse tópico identificaram 24 soluções diferentes para os problemas existente no ensino de Programação.

As metodologias ativas consideradas neste estudo incluem: aprendizagem baseada em problemas, aprendizagem baseada em desempenho, aprendizagem baseada em projetos, aprendizagem baseada em equipes e sala de aula invertida. A colaboração incluí a revisão por pares e a solução de multimídia incluem módulos educacionais, recursos áudio/visual, tais como filmes, animações, slides, gráficos, textos, Moodle, dentre outros. Os ambientes de programação contemplam diversas linguagens de programação como Java, Python, $\mathrm{C}$, $\mathrm{C}++$ e linguagens de programação visual como Scratch, AppInventor e BeeBot.

\section{Limitações}

Após finalizar o estudo terciário, levantaram-se as limitações existentes deste estudo. Nessa perspectiva, acredita-se que existem duas limitações que podem ser consideradas neste traDOI: 
balho. A primeira foi constatada a partir dos resultados obtidos ao longo da condução do estudo terciário, em que foram observados que os estudos secundários também incluíram conferências e periódicos brasileiros de temáticas específicas (e.g., SBGames). Neste estudo, foram considerados somente estudos providos de conferências e periódicos que relacionam a educação em computação com as tecnologias educacionais, por isso não foram incluídas essas conferências. Por outro lado, é possível que conferências como SBGames, Simpósio Brasileiro de Sistemas de Informação e outras possam ter publicado estudos secundários relevantes. Além dessa limitação, este estudo não incluiu estudos secundários socializados em veículos de divulgação científica internacionais. Portanto, trabalhos produzidos por pesquisadores do Brasil que foram divulgados em veículos internacionais não foram considerados.

\section{Conclusões}

A partir das evidências coletadas neste estudo terciário, pôde-se constatar que apesar de existirem estudos secundários que investigam tópicos específicos de computação, a grande maioria não identifica os problemas que permeiam o ensino dessas temáticas. Mesmo aqueles estudos que apresentam problemas, eles não oferecem uma relação entre as soluções existentes com os problemas identificados. Além disso, observou-se uma carência de estudos secundários que investigam o panorama existente sobre o ensino de outros tópicos relevantes em Computação como Sistemas Operacionais, Inteligência Artificial, Sistemas Distribuídos, Teoria da Computação, Análise e Projeto de Algoritmos, entre outros. Em outra perspectiva, há também uma escassez de trabalhos que sintetizam contribuições referentes aos movimentos que visam despertar o interesse de meninas para a área de computação. Portanto, fica clara a necessidade de mais estudos aprofundados na área de ensino de computação, visando reunir o conhecimento existente na comunidade de pesquisa, funcionando como fonte de informação para educadores e pesquisadores, guiando novas pesquisas que solucionem problemas ainda latentes na área.

\section{Agradecimentos}

Os autores gostariam de agradecer a CAPES - Código de Financiamento 001, ao CNPq pelo apoio financeiro e a PROPP/UFGD - SIGProj $\mathrm{n}^{\mathrm{o}} 322855.1174 .8276 .11032019$.

\section{Referências}

ARMANDA, M. d. A.; RODRIGUES, R. L.; GARCIA, V. C. Um mapeamento sistemático para problem based learning aplicado à ciência da computação. In: Workshop de Informática na Escola, 2012. p. 1-10.

BRACKMANN, C. P. Desenvolvimento do pensamento computacional através de atividades desplugadas na educação básica. Tese (Doutorado) — UFRGS, 2017.

HAO, Q. et al. A systematic investigation of replications in computing education research. ACM Transactions on Computing Education, v. 19, n. 4, p. 42-1:42-18, 2019.

HOLANDA, W. D. de; FREIRE, L. de P.; COUTINHO, J. C. da S. Estratégias de ensinoaprendizagem de programação introdutória no ensino superior: uma revisão sistemática da literatura. RENOTE, v. 17, n. 1, p. 527-536, 2019.

KITCHENHAM, B. A. et al. Systematic literature reviews in software engineering - a tertiary studdy. Information and Software Technology, v. 52, n. 8, p. 792-805, 2010

DOI: 
LIMA, J. V. et al. As metodologias ativas e o ensino em engenharia de software: uma revisão sistemática da literatura. In: Workshop de Informática na Escola, 2019. p. 1014.

RICHTER, C. J.; BERNARDI, G.; CORDENONSI, A. Z. O ensino de programação mediado por tecnologias educacionais: uma revisão sistemática de literatura. RENOTE, v. 17, n. 1, p. 517-526, 2019.

RIDEL, D. et al. Technovation hackday @ icmc-usp um instrumento de difusão e articulação de meninas na computação. In: Women in Information Technology, 2018. p. 1-5.

RODRIGUES, S.; ARANHA, E.; SILVA, T. R. Computaçao desplugada no ensino de programaçao: Uma revisao sistemática da literatura. In: Simpósio Brasileiro de Informática na Educação, 2018. p. 417.

SOUZA, D. M.; BATISTA, M. H. da S.; BARBOSA, E. F. Problemas e dificuldades no ensino de programaçao: Um mapeamento sistemático. Revista Brasileira de Informática na Educação, v. 24, n. 1, p. 39, 2016.

TRIDICO, S. et al. Ações para a inclusão feminina na era digital: Despertando o interesse em programação. In: Latin American Computing Conference, 2018. p. 1-10.

ZHANG, L.; NOURI, J. A systematic review of learning computational thinking through scratch in k-9. Computers \& Education, v. 141, p. 103607, 2019. 